interest to compare our results with those of Kolhorster' ${ }^{1}$ as the 'field' of our apparatus was similar to his. Kolhorster found for the same hours an intensity surplus of $1 \cdot 76 \pm 0 \cdot 28$ per cent, and interpreted it, with reservation, as an effect of the Nova Herculis. The two results, between the limit of error, can be said to be equal, notwithstanding the fact that

\begin{tabular}{|c|c|c|}
\hline $\begin{array}{c}\text { Interval } \\
(M . E . T .)\end{array}$ & $\begin{array}{l}\text { Intensity in } \\
\text { coincidences } \\
\text { per minute }\end{array}$ & Increase \\
\hline $8^{n}-16^{n}$ & $73 \cdot 78 \pm 0 \cdot 10$ & $1 \cdot 37 \pm 0 \cdot 17$ per cent \\
\hline $16^{\mathrm{b}}-24^{\mathrm{n}}$ & \multirow{2}{*}{$72 \cdot 78 \pm 0 \cdot 07$} & \\
\hline $0^{h}-8^{h}$ & & \\
\hline
\end{tabular}

in our case, contrary to Kolhörster's, the Nova was not at that time in the 'field' of the apparatus. When the Nova, with an average magnitude of 7 in August, was in the 'field' of the apparatus, we found the intensity $1 \cdot 84 \pm 0 \cdot 13$ per cent less than the average during other hours of the day (Fig. 1).

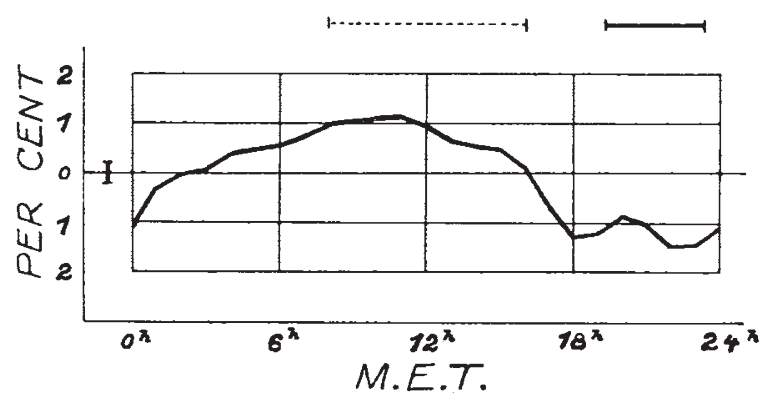

FIG. 1. Percentage variation of cosmic radiation with M.E.T. The points do not represent the original hourly values, but to smooth the curve the fourth means are plotted. Above the graph the time is marked when the Nova passed through the 'fleld' of our apparatus (July 1935, full line) and through Kolhörster's (Dec. 1934, dotted line).

It seems to be evident that the supposition expressed in an earlier paper ${ }^{3}$ is justified, and that the intensity surplus observed by Kolhörster is really nothing else but the daily intensity variation of the cosmic radiation. The real cause of this daily variation, and the reason why it is larger with countertube methods than with ionisation chamber methods, cannot yet be satisfactorily explained. We believe, however, that it is not caused by the influence of the sun, but it may be the consequence of the daily variation of the earth's magnetic field intensity, perhaps diminished by the daily variation of the outer temperature. To support this opinion we may say that we have found a correlation coefficient of -0.73 between hourly means of the cosmic ray intensity and horizontal intensity of the earth's magnetic field 4 , and again a coefficient of -0.67 between daily averages of cosmic ray intensity and outer temperature.

The experimental part of this work was kindly aided by the Hungarian Council for Natural Sciences. J. BARNóthy. M. FoRRó.

Institute of Experimental Physics, University, Budapest. Sept. 6 .

1 W. Kolhörster, Z. Phys, 93, 429; 1935

$2 \mathrm{~J}$. Barnóthy and M. Forró, NATURE, 135, 618; 1935

3 J. Barnothy and M. Forro, Z. Phys., 94, 773; 1935.

1 The magnetic data were taken from Landolt-Börnstein Erg. Bd. 3 ; they represent the mean values of the intensity for July and August in the period 1921-1930.

\section{Positive and Negative Ions in the Primary Cosmic Radiation}

THE observed east-west asymmetry of the cosmic radiation, and its increase with increasing altitude, has been interpreted in terms of an unbalanced primary radiation which is positively charged. It has been suggested by Compton and Bethe that the radiation probably consists of protons, the rest of the primary radiation consisting of equal numbers of positrons and electrons.

The existence of such an unbalanced positively charged radiation would mean that there would be a preponderance of ions of one sign in interstellar space, and, as has been pointed out by Swann ${ }^{1}$, this would give rise to enormous differences of potentials between points separated by distances which are relatively small according to astronomical standards. That such differences of potential do not exist is guaranteed by the observed state of rest of the interstellar ionised calcium clouds as shown by Ross Gunn ${ }^{2}$. These considerations suggest strongly that equal numbers of positive and negative charges exist in the cosmic ray beam.

Swann ${ }^{1}$ has shown further that any attempt to consider the emission of a greater number of ions of one sign than the other from stars or other heavenly bodies results in a condition in which the particles become bound to their. respective stars as a sort of space charge.

Similarly, it would appear that if the earth had been bombarded, since its formation, by a primary cosmic radiation in which positively charged particles predominated, it would have gradually acquired a high positive potential. Finally it would, therefore, tend to repel those ions which were positively charged, while attracting those whose charges were negative. This would result in a gradual reduction of the earth's potential until equal numbers of positive and negative charges would be incident on the earth. It is apparent, therefore, that equal numbers of positive and negative ions must be falling on the earth at any instant, and it must be concluded that the azimuthal asymmetry must be explained in some different way other than in terms of an unbalanced positive component.

Ross Gunn has suggested that the positive and negative ions travel through space with equal velocities, and that the asymmetry is due to the differences between the penetraiting power of the positive and negative ions, and to the different spirals, which, on account of their different rest masses, these particles describe in the earth's magnetic field.

Radiation Laboratory,

University of California, Berkeley. Sept. 5 .

Swann, Phys. Rev., 44, 124; 1933.

2 Ross Gunn, Phys. Rev., 45, $900 ; 1934$.

H. J. WaLke.

\section{Slow Neutrons}

For some months past we have been investigating the influence of the temperature of hydrogen containing material on the activity of silver irradiated by slow neutrons. In our experiments we have cooled down paraffin wax to the temperature of liquid air. Under these conditions, no increase of activity of silver has been observed. Moon and 